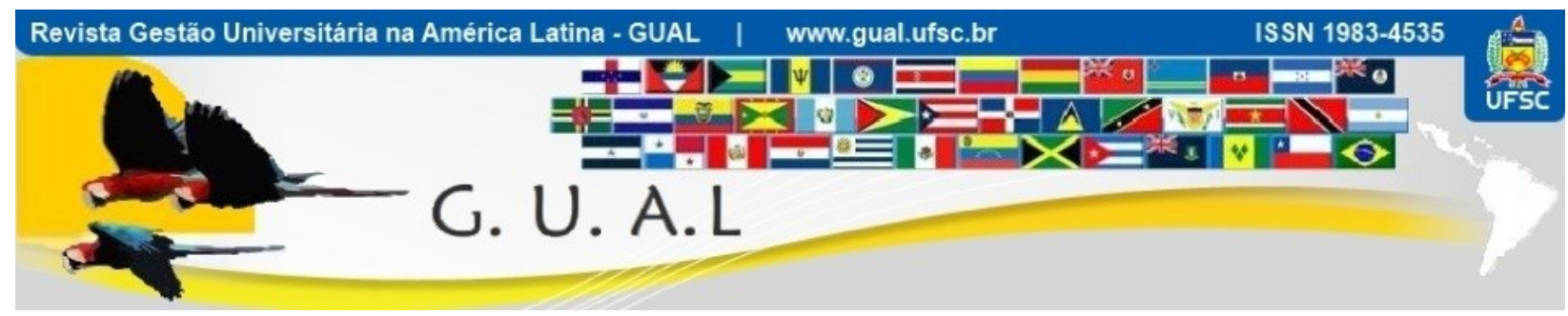

DOI: http://dx.doi.org/10.5007/1983-4535.2014v7n1p106

\title{
GESTÃO ESTRATÉGICA EM INSTITUIÇÕES DE ENSINO SUPERIOR: MAPEAMENTO DAS PUBLICAÇÕES CIENTÍFICAS NO PERÍODO DE 1997 A 2012
}

\section{STRATEGIC MANAGEMENT IN HIGHER EDUCATION INSTITUTIONS: MAPPING OF SCIENTIFIC PUBLICATIONS IN THE PERIOD 1997 TO 2012}

Osvair Almeida Matos, Mestrando Universidade do Sul de Santa Catarina - UNISUL osvair@sc.senai.br

Daniella Macedo Venâncio, Mestre Universidade do Sul de Santa Catarina - UNISUL dani@fuzz.com.br

Ademar Dutra, Doutor Universidade do Sul de Santa Catarina - UNISUL ademar.unisul@gmail.com

Recebido em 23/agosto/2013

Aprovado em 24/novembro/2013

Sistema de Avaliação: Double Blind Review

Esta obra está sob uma Licença Creative Commons Atribuição-Uso. 


\title{
RESUMO
}

Este artigo analisou a produção científica sobre a gestão estratégica em Instituições de Ensino Superior publicada nos anais dos eventos acadêmicos da Associação Nacional de PósGraduação em Administração (ANPAD) e na base de dados Scientific Electronic Library Online (SciELO) no período de 1997 a 2012. Neste sentido, foram identificados e analisados os seguintes indicadores bibliométricos: (i) distribuição por artigos pesquisados, (ii) amostra selecionada, (iii) fonte de coleta, (iv) palavras-chave, (v) ano de publicação, (vi) autores mais prolíficos, (vii) instituições mais prolíficas e (viii) estado. Trata-se de uma pesquisa de natureza exploratória, cuja abordagem é descritiva, envolvendo análise documental e abordagem quantitativa. A amostra compõe-se de 175 artigos, que, após a aplicação dos procedimentos de análise, resultou em 18 publicações selecionadas. Concluiu-se com este trabalho que doze (12) artigos foram localizados na base de dados da ANPAD e seis (6) na SciELO, sendo que 50\% das publicações aconteceram no período de 2010 a 2012, por isso infere-se que se trata de um tema novo. Constatou-se também que as Universidades PUC/RS, PUC/PR e UFPR são as mais prolíficas, com 33\% das publicações, e que $61 \%$ dos artigos estão publicados nos Estados do Paraná, do Rio Grande do Sul e de São Paulo.

Palavras-chave: Gestão Estratégica. Instituições de Ensino. Planejamento Estratégico.

\begin{abstract}
This article was to review the scientific literature on strategic management in higher education institutions published in the annals of academic events of the National Association of Graduate Business Administration (ANPAD) and database Scientific Electronic Library Online (SciELO) from 1997 to 2012. In this sense, we identified and analyzed the following bibliometric indicators: (i) distribution articles surveyed, (ii) selected sample, (iii) source collection, (iv) keywords, (v) publication year, (vi) most prolific authors, (vii) the most prolific institutions and (viii) state. The study consists of an exploratory study, which is descriptive approach, involving documentary analysis and quantitative approach. The sample consists of 175 articles, which, after applying analytical procedures resulted in 18 publications selected. The conclusion of this work than twelve (12) items were found in the database ANPAD six (6) in SciELO, and 50\% of the publications occurred in the period from 2010 to 2012, so it appears that it is a new theme. It was also found that universities PUC / RS, PUC / PR and UFPR are the most prolific, with $33 \%$ of publications and $61 \%$ of the articles are published in the states of Paraná, Rio Grande do Sul and São Paulo.
\end{abstract}

Keywords: Strategic Management. Education Institutions. Strategic Planning. 


\section{INTRODUÇÃO}

A velocidade com que as mudanças econômicas, sociais e culturais ocorrem na sociedade atual exige atenção das Instituições de Ensino Superior (IES), uma vez que estas têm o papel fundamental de formar cidadãos que gerem novos conhecimentos e, consequentemente, o desenvolvimento de toda a sociedade (CAMPOS; VERNI, 2006).

Historicamente, a primeira universidade brasileira foi fundada no Rio de Janeiro, em 1920 (Stallivieri, 2003). Contudo, em 2010, de acordo com o censo da educação superior realizado anualmente pelo Instituto Nacional de Estudos e Pesquisas Educacionais Anísio Teixeira (INEP) - foram oferecidos 29.507 cursos de formação superior, incluindo os cursos de graduação e sequenciais de formação específica, presenciais e a distância (INEP, 2011).

Percebe-se um número expressivo de cursos de graduação oferecidos no Brasil. Esse cenário tende a promover a competição entre as IES, o que de modo geral indica a necessidade das IES se tornarem mais preparadas para atrair e reter alunos, professores e demais funcionários. Neste caso, faz-se necessário um comportamento distinto no que se refere às decisões estratégicas (MARCO, 2011).

Para compreender como as IES se mobilizam estrategicamente, é fundamental investigar informações referentes às pesquisas que buscam estudar o impacto da gestão estratégica nas IES. Desta forma, utiliza-se o estudo bibliométrico, que se propõe a analisar as fontes utilizadas no levantamento bibliográfico, ou seja, ele analisa a produção científica de determinada área ou temática observando e examinando a literatura que subsidiou os pesquisadores no desenvolvimento de seus trabalhos. Desta forma, é possível levantar especificidades da literatura usada no processo de construção do conhecimento e obter indicadores que possibilitem uma avaliação do estágio de maturidade da produção científica de certa área ou programa de pesquisa (HERNANDES; LOMBARDI, 2011, p. 3).

Neste sentido, este estudo visa analisar os artigos publicados nos anais dos eventos acadêmicos da Associação Nacional de Pós-Graduação em Administração (ANPAD) e nos periódicos da Scientific Electronic Library Online (SciELO), no período de 2007 a 2012, referentes à produção e à disseminação do conhecimento científico sobre a gestão estratégica em Instituições Educacionais. Para isto, foram propostos como objetivos os seguintes indicadores bibliométricos: (1) identificar a distribuição por artigos pesquisados, (2) definir a amostra, (3) definir a fonte de coleta, (4) definir as palavras-chave, (5) identificar o ano das publicações, (6) identificar os autores mais prolíficos, (7) identificar as instituições mais 
prolíficas e (8) identificar os estados mais prolíficos. O estudo será delimitado na relação entre a gestão e o planejamento estratégico em Instituições de Ensino. A justificativa do tema se dá pelo fato de que o desempenho das organizações está associado à capacidade dos gestores de formarem, implantarem e acompanharem as estratégias que as levem a apresentar desempenho superior diante de seus concorrentes.

\section{FUNDAMENTAÇÃO TEÓRICA}

A estruturação da fundamentação teórica abrange definições de gestão estratégica, planejamento estratégico e estudos bibliométricos.

\subsection{PLANEJAMENTO ESTRATÉGICO}

A compreensão da estratégia privilegia a dinâmica dos resultados, sobretudo nos processos de mudança e inovação, nas gestões de informação e conhecimento e na aprendizagem organizacional, destacando-se o papel do capital humano e das alianças empresariais na consolidação de um processo de administração estratégica dinâmico, complexo, integrado e de melhoria de resultados (YOSHIMO, 1997). Para Junior (2003), a gestão estratégica encontra-se não no trabalho realizado em departamentos isolados, mas sim por processos, tornando as atividades organizacionais integradas, sistêmicas e interdependentes.

De acordo com Santos (2008), o conceito de estratégia teve sua origem presumidamente no século XI a. C., na China antiga, por meio das contribuições pioneiras de generais e estrategistas militares, porém, os desenvolvimentos teóricos sobre a gestão estratégica se consolidaram somente a partir da década de 1960. Entretanto, de acordo com Hernandes e Lombardi (2011), não há uma definição universalmente aceita para este significante, Estratégia é uma palavra usualmente empregada no meio empresarial e sua significação é compreendida intuitiva e contextualmente por não existir nenhuma definição unanimemente reconhecida.

A vasta literatura não imprime um conceito único para o planejamento estratégico. Entretanto, de acordo com o dicionário Houaiss, planejar significa ter a intenção de, organizar plano ou roteiro, programar (HOUAISS, 2009). Já no ambiente organizacional, planejar exprime a ideia de preestabelecer ações futuras para alcançar objetivos desejados, sendo que, 
para isso, a utilização de metas específicas e mensuráveis, com prazos estabelecidos, é muito comum (SILVEIRA; BASTIAS, 2003).

Estrada et al., (2007) afirmam que o planejamento estratégico evoluiu nos últimos tempos, porém ainda é alvo de críticas, especialmente no que se refere à sua implementação. Os autores supracitados sintetizam algumas análises apontadas por pesquisadores como Mintzberg (1983, 1985, 1992, 2000), Hamel e Prahalad (1995), Hussey (1998) e Robbins (2001), tais como:

- O planejamento estratégico tende a criar rigidez, limitando a organização em metas específicas e com prazos determinados;

- O foco em metas específicas não admite a presença do imprevisível, indo de encontro ao ambiente dinâmico, mutável e imprevisível que se apresenta no mundo atual;

- O planejamento é criado de acordo como o presente, muitas vezes é impossível antever problemas ou oportunidades futuras;

- As organizações tendem a repetir ações que geraram sucesso no passado, fato este que pode gerar excesso de confiança e cegueira com relação a dificuldades futuras;

- O planejamento formal por vezes descuida de aspectos culturais e organizacionais.

No entanto, apesar das críticas, o processo de planejar é necessário e se faz presente em grande parte das organizações. Estudiosos buscam corrigir os erros e transformar essa ferramenta em algo que possa auxiliar os gestores na administração de seus negócios. Isso vem sendo feito por meio de pesquisas acadêmicas e aplicações empíricas, chegando a um modelo que se denomina Gestão Estratégica (ESTRADA et al., 2007).

\subsection{GESTÃO ESTRATÉGICA}

A literatura sobre gestão estratégica define estratégia como um conceito multifacetado, com diferentes dimensões, tais como: pensamento estratégico, processo estratégico e mudança estratégica (JR. et al., 2012). A transformação de intenções em ações, em especial, tem se mostrado um grande desafio para os gestores organizacionais. Contudo, tais dimensões possuem entre elas dois pontos convergentes, o primeiro diz respeito ao fato da gestão estratégica estar ligada a uma preocupação com o futuro da empresa; o segundo, é que na gestão estratégia a atenção é dirigida para a formulação e para a implementação (NICOLAU, 2001). 


\section{GESTÃO ESTRATÉGICA EM INSTITUIÇÕES DE ENSINO SUPERIOR: MAPEAMENTO DAS \\ PUBLICAÇÕES CIENTÍFICAS NO PERÍODO DE 1997 A 2012 \\ DOI: http://dx.doi.org/10.5007/1983-4535.2014v7n1p106}

Para Chandler (1962), a estratégia é a fixação de metas e a criação de caminhos para se alcançar os objetivos traçados. Já para Ansoff (1965), trata-se de um processo em que se faz necessária a tomada de decisão sem conhecer o futuro. Nesse sentido, Mintzberg (1988) afirma que, para uma tomada de decisão eficaz, é preciso levar em consideração o meio envolvente, e o autor reconhece que a formação da estratégia pode ser deliberada ou emergente. As estratégias deliberadas são aquelas planejadas com antecedência; as emergentes são aquelas que emergem no dia a dia das organizações e que algumas vezes impõem a necessidade de reorganização do planejamento estratégico formal.

Corroborando, Júnior e Castro (2006) afirmam que a gestão estratégica se refere ao empenho da organização em formular estratégias que guiarão o caminho da organização, considerando para isso não somente o processo formal de elaboração das estratégias, mas um processo que leva em consideração a identificação dos conhecimentos essenciais para a empresa, como o processo de aprendizagem individual e organizacional e o aproveitamento das competências individuais e organizacionais, visando à criação de vantagem competitiva.

Para compreender os processos que dão origem às estratégias, ou seja, a forma como estas surgem e se implementam, Nicolau (2001) propõe que é importante ter consciência de que esse não é um processo idêntico em todas as organizações, uma vez que há um conjunto de fatores externos (características e condições do meio-ambiente) e de condições internas (dimensão, capacidades materiais, humanas e organizacionais) que forma uma conjuntura particular em cada caso. A autora então apresenta três linhas para a análise da formação das estratégias: (i) a formação de estratégia como um processo formal, (ii) a formação da estratégia como um processo negociado e (iii) a formação da estratégia como um processo de construção permanente.

Segundo Nicolau (2001), no processo formal de formação estratégica, o principal instrumento é o planejamento estratégico, já que este proporciona uma estrutura unificada que facilitará a identificação dos problemas da organização por parte dos gestores bem como a identificação de novas oportunidades, ou seja, forças a serem potencializadas e fraquezas a serem cuidadas. Com relação à formação de estratégia como um processo negociado, a organização é compreendida pelos gestores como um corpo social e não apenas com uma unidade técnico-econômica e, por essa razão, o autor destaca a importância da inserção da dimensão social e política no processo. Já o processo de construção permanente admite o meio envolvente como um fator imprevisível, por isso, a estratégia não se desenvolve por 
meio de processos claros, ordenados e controlados. No processo de formação de estratégia permanente, as ações se desencadeiam de tal forma que não se podem conceber suas implicações no futuro, nem tampouco reconhecer seu ponto de partida.

A gestão estratégia de uma organização pode conter tanto o esforço de racionalização e de planejamento formal quanto um processo de negociação com atores internos e externos. Entretanto, a flexibilidade se faz necessária durante todo o processo de gestão, para que não se despreze o processo de aprendizagem gerado pelos agentes, a partir da interpretação que eles fazem de situações que emergem do ambiente interno e externo à organização (REBELO et al., 2007).

\subsection{ESTUDOS BIBLIOMÉTRICOS}

De acordo com Vanti (2002), embora o termo bibliometria tenha sido cunhado por Alan Pritchard, em 1969, quem realmente utilizou pela primeira vez este termo foi Paul Otlet, várias décadas antes, em sua obra intitulada Traité de documentatión, de 1934. Entretanto, Pritchard popularizou o uso da palavra 'bibliometria' quando sugeriu que esta deveria substituir o termo 'bibliografia estatística', que vinha sendo utilizado desde a menção feita em 1922 por Edward Wyndham Hulme em uma conferência na Universidade de Cambridge, reportando-se a um estudo pioneiro de Cole \& Eales, de 1917, referente à análise estatística de uma bibliografia de Anatomia Comparada.

Alvarenga (1998) assinala que a bibliometria é um ramo da ciência da informação que costuma priorizar a produção científica convencional como objeto, embora não restrinja seu universo de pesquisa apenas aos domínios desse tipo de literatura. Corroborando, Araújo (2006, p. 12) afirma que a bibliometria consiste "na aplicação de técnicas estatísticas e matemáticas para descrever aspectos da literatura e outros meios de comunicação (análise quantitativa da informação)".

\subsubsection{Estudos similares}

Dentro da literatura não foi encontrado nenhum estudo bibliométrico que investigasse a produção científica sobre formação de estratégia nas IES. Entretanto, constataram-se alguns estudos bibliométricos que investigam outras questões relacionadas com as IES, citando estudos que analisaram as produções acadêmicas em áreas específicas como administração (LORDSLEEM, 2009; CORREAA, 2012), contabilidade (ESPARTEL, 2011; FILHO, 2012), 
educação especial (PIZZANI et al., 2010; SILVA et al., 2013) e educação a distância (CARPES, 2010; KNEIPP, 2012). No que tange à gestão estratégica, foram encontrados dois estudos bibliométricos, um investigando artigos que enfatizam a estratégia de negócios internacionais (PINTO, 2010) e outro que analisa a gestão estratégia em pequenas e médias empresas (ROCHA, 2011).

No que tange aos estudos bibliométricos que investigaram questões relacionadas às IES, pode-se constatar duas convergências entre eles, a primeira refere-se à evolução da pesquisa, ou seja, as produções científicas sobre IES vêm aumentando nos últimos anos, em especial a partir de 2006 (CARPES, 2010; Corrêa, 2012; FILHO, 2012; SILVA et al., 2013), a segunda trata-se do fato de que apresentam a região sul e sudeste como as mais prolixas (LORDSLEEM, 2009; PIZZANI et al., 2010; CARPES, 2010; Corrêa, 2012; FILHO, 2012; SILVA et al., 2013). O fato de não ter encontrado outro estudo bibliométrico que analisasse artigo(s) sobre gestão estratégica das IES impedirá a comparação dos achados desta pesquisa.

\section{METODOLOGIA}

No que tange à natureza do objetivo, a presente pesquisa é classificada como descritiva, pois identifica e descreve os artigos publicados nos anais dos eventos acadêmicos da Associação Nacional de Pós-Graduação em Administração (ANPAD) e nos periódicos da Scientific Electronic Library Online (SciELO) referentes à produção e à disseminação do conhecimento científico sobre a gestão estratégica em instituições educacionais (COLLIS; HUSSEY, 2005).

Com o intuito de alcançar o objetivo proposto, optou-se pela bibliometria como estratégia de coleta, refinamento e análise dos dados. Macias-Chapula (1998, p. 134) cita que a bibliometria "é o estudo dos aspectos quantitativos da produção, disseminação e uso da informação registrada", segundo Cunha et al. (2011, p. 58), a bibliometria também pode ser definida como: um estudo quantitativo das unidades bibliográficas publicadas; a aplicação de métodos estatísticos e matemáticos ao estudo do uso dos livros e outros meios; o estudo quantitativo da produção de documentos.

Em relação à abordagem do problema, a pesquisa caracteriza-se como quantitativa, pois a análise realizada procura identificar características da produção científica e utiliza meios estatísticos para tabular os dados e informar os resultados. Segundo Richardson (1999), os estudos qualitativos caracterizam-se pelo não emprego de instrumental estatístico no 
processo de análise de um problema, o autor destaca que os quantitativos empregam instrumentos estatísticos, tanto na coleta quanto no tratamento dos dados.

Quanto à coleta de dados, (a) utilizou-se como critério de escolha os artigos que apresentassem os termos "Instituições de Ensino Superior", "Gestão Estratégica" e "Planejamento Estratégico" no título, no resumo ou nas palavras-chave; (b) a população alvo é constituída pelos sítios da Associação Nacional de Pós-Graduação em Administração (ANPAD) e da Scientific Electronic Library Online (SciELO); (c) examinaram-se a distribuição por artigos pesquisados, amostra selecionada, fonte de coleta, palavras-chave, ano de publicação, autores mais prolíficos, instituições mais prolíficas, estado; (d) foram coletados todos os artigos publicados pelos periódicos compreendidos entre os anos de 1997 a 2012.

\subsection{PROCEDIMENTOS DA PESQUISA}

Para analisar a produção científica referente à gestão estratégica em instituições educacionais, foi adotada metodologia composta por cinco etapas: 1. Definição do tema de pesquisa, objetivos e indicadores bibliométricos; 2. Pesquisa dos dados; 3. Coleta de dados; 4. Organização e tratamento bibliométrico dos dados coletados; 5. Análise dos resultados. A Figura 1 representa todas as etapas do procedimento de pesquisa.

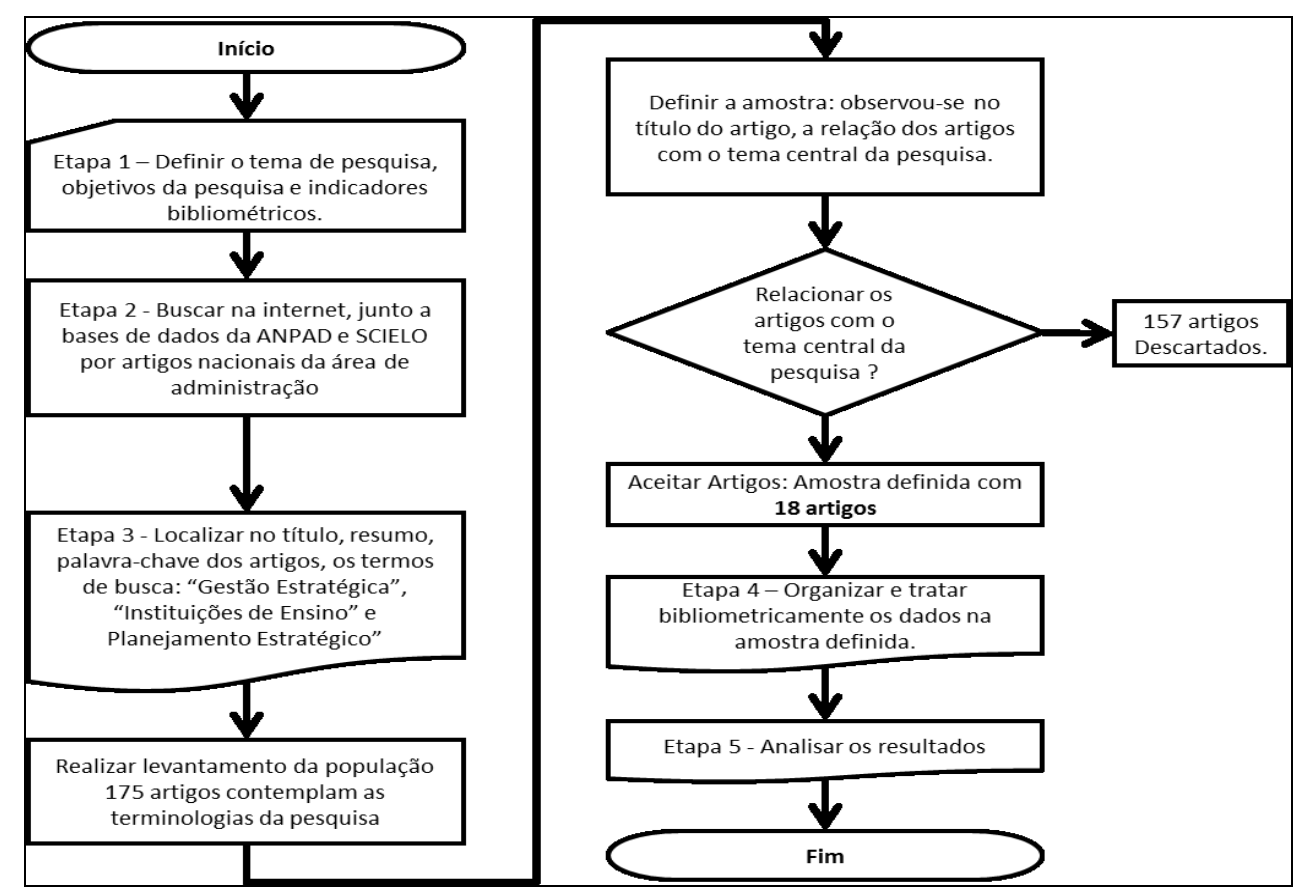

Figura 1 Fluxograma - Procedimentos da pesquisa.

Fonte: Elaborado pelos autores (2012). 


\section{GESTÃO ESTRATÉGICA EM INSTITUIÇÕES DE ENSINO SUPERIOR: MAPEAMENTO DAS \\ PUBLICAÇÕES CIENTÍFICAS NO PERÍODO DE 1997 A 2012 \\ DOI: http://dx.doi.org/10.5007/1983-4535.2014v7n1p106}

A primeira etapa aconteceu no período de 12/03/2012 a 19/03/2012. Nesta etapa, definiu-se o seguinte tema de pesquisa "Gestão Estratégica em Instituições Educacionais", cujo objetivo foi identificar e quantificar as informações referentes à produção e à disseminação do conhecimento científico publicado em eventos e periódicos científicos da área da administração referente à gestão estratégica em instituições educacionais. Neste período foram estruturados pelos pesquisadores os seguintes indicadores bibliométricos para análise: (i) distribuição por artigos pesquisados, (ii) amostra selecionada, (iii) fonte de coleta, (iv) palavras-chave, (v) ano de publicação, (vi) autores mais prolíficos, (vii) instituições mais prolíficas, (viii) estado.

A pesquisa dos dados foi definida pelos pesquisadores no período de 19/03/2012 a 26/03/2012. Buscou-se, nesta etapa, identificar eventos e periódicos disponíveis em meio digital, o que contribuiria para um êxito da pesquisa. Desta forma, optou-se por fazer a pesquisa nas publicações dos eventos acadêmicos da Associação Nacional de Pós-Graduação em Administração (ANPAD) e nos periódicos da Scientific Electronic Library Online (SciELO).

A coleta de dados foi realizada entre 2 de abril e 15 de junho de 2012, nos sítios eletrônicos da ANPAD e SciELO. Foram realizadas buscas por meio das palavras-chave "Gestão Estratégica", "Instituições de Ensino" e "Planejamento Estratégico" e foram encontrados 175 artigos publicados no período de 1997 a 2012, conforme se observa na Tabela 1.

Tabela 1 Artigos localizados na Anpad e Scielo no período de 1997 a 2012.

\begin{tabular}{|c|c|c|}
\hline ORDEM & EVENTO/PERIÓDICOS & $\begin{array}{l}\text { NÚMERO DE } \\
\text { ARTIGOS }\end{array}$ \\
\hline 1 & Encontro da ANPAD - EnANPAD & 78 \\
\hline 2 & Encontro de Estudos em Estratégia - 3Es & 16 \\
\hline 3 & Revista de Administração Contemporânea (RAC) & 13 \\
\hline 4 & Revista de Administração Pública (RAP) & 6 \\
\hline 5 & $\begin{array}{l}\text { Encontro de Ensino e Pesquisa em Administração e Contabilidade - } \\
\text { EnEPQ }\end{array}$ & 5 \\
\hline 6 & Produção & 5 \\
\hline 7 & SIMPÓSIO & 5 \\
\hline 8 & Gestão \& Produção & 4 \\
\hline 9 & Cadernos EBAPE.BR & 3 \\
\hline 10 & Ciência \& Saúde Coletiva & 3 \\
\hline 11 & Encontro de Administração Pública e Governança - EnAPG & 3 \\
\hline 12 & RAE eletrônica & 3 \\
\hline 13 & Revista Administração de Empresas (RAE) & 3 \\
\hline 14 & Texto \& Contexto - Enfermagem & 3 \\
\hline
\end{tabular}


5 Cadernos de Saúde Pública

16 Encontro de Marketing - EMA

17 EnGPR

18 REM: Revista Escola de Minas

19 Revista Brasileira de Psiquiatria

20 Avaliação: Revista da Avaliação da Educação Superior (Campinas)

21 Ciência da Informação

22 Educação \& Sociedade

23 Encontro de Administração da Informação - EnADI 2009

24 EnEO

25 Ensaio: Avaliação e Políticas Públicas em Educação

26 Interações

27 Interface - Comunicação, Saúde, Educação

28 JISTEM - Journal of Information Systems and Technology

Management

29 Perspectivas em Ciência da Informação

30 Psico-USF

31 RAM. Revista de Administração Mackenzie

32 Revista Brasileira de Enfermagem

33 Revista Contabilidade \& Finanças

34 Revista de Saúde Pública

Total

\begin{tabular}{c}
\hline 2 \\
\hline 2 \\
\hline 2 \\
\hline 2 \\
\hline 2 \\
\hline 1 \\
\hline 1 \\
\hline 1 \\
\hline 1 \\
\hline 1 \\
\hline 1 \\
\hline 1 \\
\hline 1
\end{tabular}

Fonte: Elaborada pelos autores (2012).

Dos artigos identificados, observa-se que 71,42\% (125) foram publicados no período de 2006 a 2011, conforme o Gráfico 1. Esta constatação demonstra uma produção recente do tema nos eventos da ANPAD e nas publicações da SciELO.

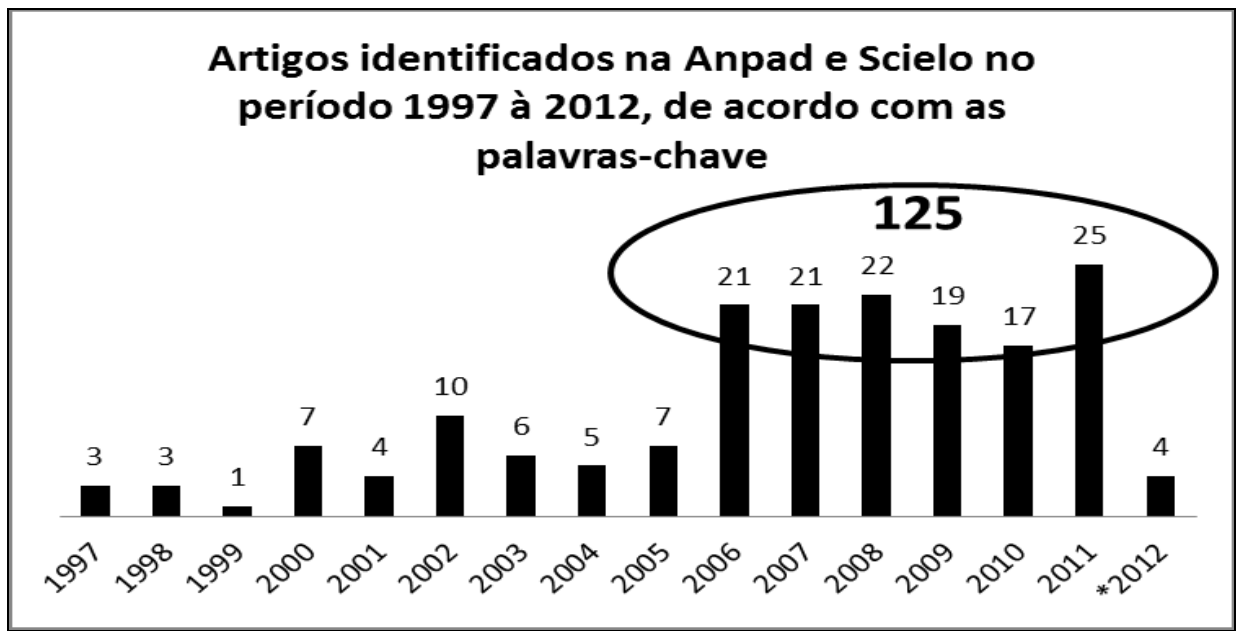

Gráfico 1 Artigos identificados nos eventos da ANPAD e nas publicações da SciELO no período de 1997 a 2012, de acordo com as palavras-chave.

Fonte: Elaborado pelos autores (2012) - Análise até 15/06/2012. 
Ao serem analisados os títulos e resumos dos artigos do Quadro 1, identificou-se que dezoito (18) deles possuíam relação direta com o tema central da pesquisa. Estes artigos foram definidos como amostra.

A seguir, foram abertos todos os arquivos identificados na amostra, coletando-se, em instrumento elaborado para os propósitos da pesquisa, o título do artigo, o estrato ao qual ele estava vinculado, as palavras-chave, os autores, as instituições de ensino dos autores, o ano de publicação do artigo, o periódico/evento associado, a natureza do estudo e a abordagem do Problema de Pesquisa. Os critérios e as variáveis desse instrumento foram compilados em formato de gráficos e tabelas.

\section{ANÁLISE DOS RESULTADOS}

Nesta seção serão apresentados os seguintes indicadores bibliométricos: (i) distribuição por artigos pesquisados, (ii) amostra selecionada, (iii) fonte de coleta, (iv) palavras-chave, (v) ano de publicação, (vi) autores mais prolíficos, (vii) instituições mais prolíficas, (viii) estado.

\subsection{DISTRIBUIÇÃO POR ARTIGOS PESQUISADOS}

O Gráfico 2 apresenta a estratificação de todos os artigos pesquisados nos sítios eletrônicos da ANPAD e da SciELO.

\section{Total de Artigos Pesquisados}

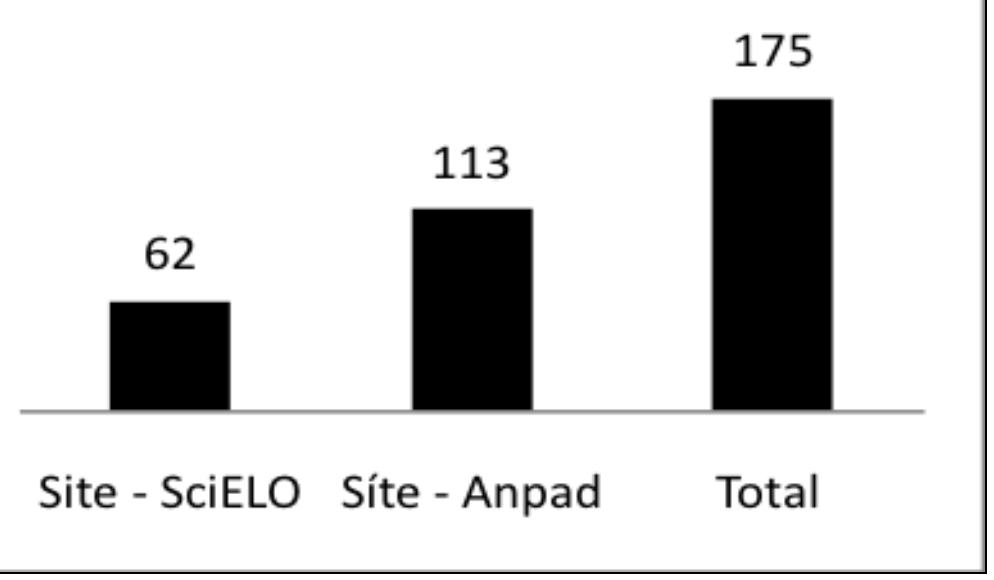

Gráfico 2 Total de artigos pesquisados na ANPAD e SciELO Fonte: Elaborado pelos autores (2012). 


\section{GESTÃO ESTRATÉGICA EM INSTITUIÇÕES DE ENSINO SUPERIOR: MAPEAMENTO DAS \\ PUBLICAÇÕES CIENTÍFICAS NO PERÍODO DE 1997 A 2012 \\ DOI: http://dx.doi.org/10.5007/1983-4535.2014v7n1p106}

Identificou-se 175 artigos. Destes, 35\% (62) foram localizados no sítio eletrônico da SciELO e 65\% (113) foram identificados no sítio eletrônico da ANPAD.

\subsection{CLASSIFICAÇÃO DA AMOSTRA DE ARTIGOS SELECIONADA}

Para a definição da amostra, levou-se em consideração a relação identificada entre o planejamento estratégico e/ou a gestão estratégica com as Instituições de Ensino. Esta relação foi verificada por meio da leitura dos resumos dos 175 artigos pesquisados. O Quadro 1 apresenta a amostra selecionada para a pesquisa bibliométrica:

\begin{tabular}{|c|c|c|c|c|}
\hline ORDEM & TÍTULO DO ARTIGO & $\begin{array}{l}\text { FONTE DE } \\
\text { COLETA }\end{array}$ & $\begin{array}{l}\text { PALAVRAS- } \\
\text { CHAVE }\end{array}$ & $\begin{array}{c}\text { ANO DE } \\
\text { PUBLICAÇÃO }\end{array}$ \\
\hline 1 & $\begin{array}{l}\text { A contribuição do planejamento estratégico } \\
\text { para o alcance de objetivos institucionais: } \\
\text { estudo de caso em uma universidade particular } \\
\text { no estado do Pará }\end{array}$ & ANPAD & $\begin{array}{l}\text { Planejamento } \\
\text { Estratégico }\end{array}$ & 2011 \\
\hline 2 & $\begin{array}{l}\text { Análise da implementação de políticas } \\
\text { públicas educacionais na Bahia: as relações } \\
\text { entre a certificação ocupacional de dirigentes } \\
\text { e o planejamento estratégico das escolas }\end{array}$ & ANPAD & $\begin{array}{l}\text { Planejamento } \\
\text { Estratégico }\end{array}$ & 2008 \\
\hline 3 & $\begin{array}{l}\text { Avaliação em movimento: do PAIUB ao PGE } \\
\text { o caso da Universidade Federal de Goiás }\end{array}$ & SciELO & $\begin{array}{c}\text { Gestão } \\
\text { Estratégica }\end{array}$ & 2010 \\
\hline 4 & $\begin{array}{l}\text { Contribuições para a gestão estratégica de } \\
\text { instituições de ciência e tecnologia }\end{array}$ & SciELO & $\begin{array}{c}\text { Gestão } \\
\text { Estratégica }\end{array}$ & 2011 \\
\hline 5 & $\begin{array}{l}\text { Elementos que influenciam o planejamento } \\
\text { estratégico em universidades: um estudo de } \\
\text { caso }\end{array}$ & ANPAD & $\begin{array}{l}\text { Planejamento } \\
\text { Estratégico }\end{array}$ & 2006 \\
\hline 6 & $\begin{array}{l}\text { Estratégia e performance organizacional em } \\
\text { Instituições de Ensino Superior: as PUCs } \\
\text { brasileiras em busca de efetividade }\end{array}$ & ANPAD & $\begin{array}{l}\text { Instituições de } \\
\text { Ensino }\end{array}$ & 2000 \\
\hline 7 & $\begin{array}{l}\text { Estratégia, liderança e clima organizacional } \\
\text { em Instituições de Ensino: contextualizando } \\
\text { conceitos para a compreensão de um setor } \\
\text { específico }\end{array}$ & ANPAD & $\begin{array}{l}\text { Instituições de } \\
\text { Ensino }\end{array}$ & 2011 \\
\hline 8 & $\begin{array}{l}\text { Gestão estratégica da Tecnologia de } \\
\text { Informação em uma Instituição de Ensino } \\
\text { como Diferencial Competitivo }\end{array}$ & ANPAD & $\begin{array}{l}\text { Gestão } \\
\text { Estratégica }\end{array}$ & 2006 \\
\hline 9 & $\begin{array}{l}\text { Gestão estratégica multicultural baseada no } \\
\text { Balanced Scorecard em Instituições de } \\
\text { Ensino. }\end{array}$ & SciELO & $\begin{array}{l}\text { Planejamento } \\
\text { Estratégico }\end{array}$ & 2012 \\
\hline 10 & $\begin{array}{l}\text { Gestão estratégica: um exame de práticas em } \\
\text { universidades privadas }\end{array}$ & SciELO & $\begin{array}{c}\text { Gestão } \\
\text { Estratégica }\end{array}$ & 2012 \\
\hline 11 & $\begin{array}{l}\text { Informações estratégicas de suporte à } \\
\text { implementação de gestão de relacionamento } \\
\text { com cliente em Instituições de Ensino } \\
\text { Superior }\end{array}$ & ANPAD & $\begin{array}{l}\text { Instituições de } \\
\text { Ensino }\end{array}$ & 2007 \\
\hline 12 & $\begin{array}{l}\text { Inteligência estratégica em Instituições de } \\
\text { Ensino Superior }\end{array}$ & SciELO & $\begin{array}{l}\text { Instituições de } \\
\text { Ensino } \\
\end{array}$ & 2010 \\
\hline 13 & $\begin{array}{l}\text { Metodologia para projeto de planejamento } \\
\text { estratégico de informações alinhado ao } \\
\text { planejamento estratégico: a experiência do } \\
\text { SENAC-PR. }\end{array}$ & SciELO & $\begin{array}{l}\text { Planejamento } \\
\text { Estratégico }\end{array}$ & 2003 \\
\hline
\end{tabular}




\section{GESTÃO ESTRATÉGICA EM INSTITUIÇÕES DE ENSINO SUPERIOR: MAPEAMENTO DAS \\ PUBLICAÇÕES CIENTÍFICAS NO PERÍODO DE 1997 A 2012 \\ DOI: http://dx.doi.org/10.5007/1983-4535.2014v7n1p106}

\begin{tabular}{|c|l|c|c|c|}
\hline 14 & $\begin{array}{l}\text { Modelos de avaliação de desempenho } \\
\text { organizacional nas Instituições de Ensino } \\
\text { Superior da região metropolitana de São Paulo }\end{array}$ & ANPAD & $\begin{array}{c}\text { Instituições de } \\
\text { Ensino }\end{array}$ & 2011 \\
\hline 15 & $\begin{array}{l}\text { Planejamento estratégico em Instituições de } \\
\text { Ensino Superior: um estudo do Curso de } \\
\text { Administração de Empresas da UNISINOS }\end{array}$ & ANPAD & $\begin{array}{c}\text { Instituições de } \\
\text { Ensino }\end{array}$ & 2001 \\
\hline 16 & $\begin{array}{l}\text { Planejamento estratégico em uma incubadora: } \\
\text { semelhanças e particularidades do processo }\end{array}$ & ANPAD & $\begin{array}{l}\text { Planejamento } \\
\text { Estratégico }\end{array}$ & 2006 \\
\hline 17 & $\begin{array}{l}\text { Planejamento estratégico em uma } \\
\text { universidade pública brasileira }\end{array}$ & ANPAD & $\begin{array}{l}\text { Planejamento } \\
\text { Estratégico } \\
\text { Gestão } \\
\text { Estratégica }\end{array}$ & 2002 \\
\hline
\end{tabular}

Quadro 1 Amostra de artigos selecionados

Fonte: Elaborado pelos autores (2012).

Observa-se no Quadro 1 os 18 artigos identificados de acordo com o tema da pesquisa. A partir desta análise, estes artigos passaram a compor a amostra pesquisada para análise bibliométrica.

\subsection{CLASSIFICAÇÃO DOS ARTIGOS POR FONTE DE COLETA}

No Tabela 2, apresenta-se a fonte de consulta dos artigos da amostra bem como os eventos e periódico de publicação:

Tabela 2 Amostra estratificada por fonte de consulta.

\begin{tabular}{|c|c|c|c|}
\hline ORDEM & FONTE & EVENTO/PERIÓDICOS & $\begin{array}{l}\text { QUANTIDADE } \\
\text { DE ARTIGOS }\end{array}$ \\
\hline 1 & http://www.anpad.org.br/ & Encontro da ANPAD - EnANPAD & 6 \\
\hline 2 & http://www.anpad.org.br/ & Encontro de Estudos em Estratégia - 3Es & 3 \\
\hline 3 & http://www.scielo.br/ & Revista Avaliação - UNICAMP & 1 \\
\hline 4 & http://www.scielo.br/ & Ciência da Informação & 1 \\
\hline & http://www.anpad.org.br/ & Encontro de Administração Pública e & \\
\hline 5 & & Governança - EnAPG & 1 \\
\hline 6 & http://www.anpad.org.br/ & $\begin{array}{l}\text { Encontro de Ensino e Pesquisa em } \\
\text { Administração e Contabilidade - EnEPQ }\end{array}$ & 1 \\
\hline & http://www.scielo.br/ & Ensaio: Avaliação e Políticas Públicas em & \\
\hline 7 & & Educação & 1 \\
\hline 8 & http://www.scielo.br/ & Perspectivas em Ciência da Informação & 1 \\
\hline 9 & http://www.scielo.br/ & Produção & 1 \\
\hline 10 & http://www.scielo.br/ & Revista de Administração Pública (RAP) & 1 \\
\hline 11 & http://www.anpad.org.br/ & SIMPÓSIO & 1 \\
\hline Total & & & 18 \\
\hline
\end{tabular}

Fonte: Elaborada pelos autores (2012).

Observa-se que 67\% (12) dos artigos da amostra foram localizados nos eventos da ANPAD e 33\% (6) na SciELO. Destaca-se que dos 12 artigos publicados na ANPAD, 50\% foram publicados no evento "Encontro da ANPAD - EnANPAD". 


\subsection{CLASSIFICAÇÃO DOS ARTIGOS POR PALAVRAS-CHAVE}

O Gráfico 3 apresenta a estratificação dos artigos da amostra pesquisada pelas palavras-chave: Instituições de Ensino, Gestão Estratégica e Planejamento Estratégico.

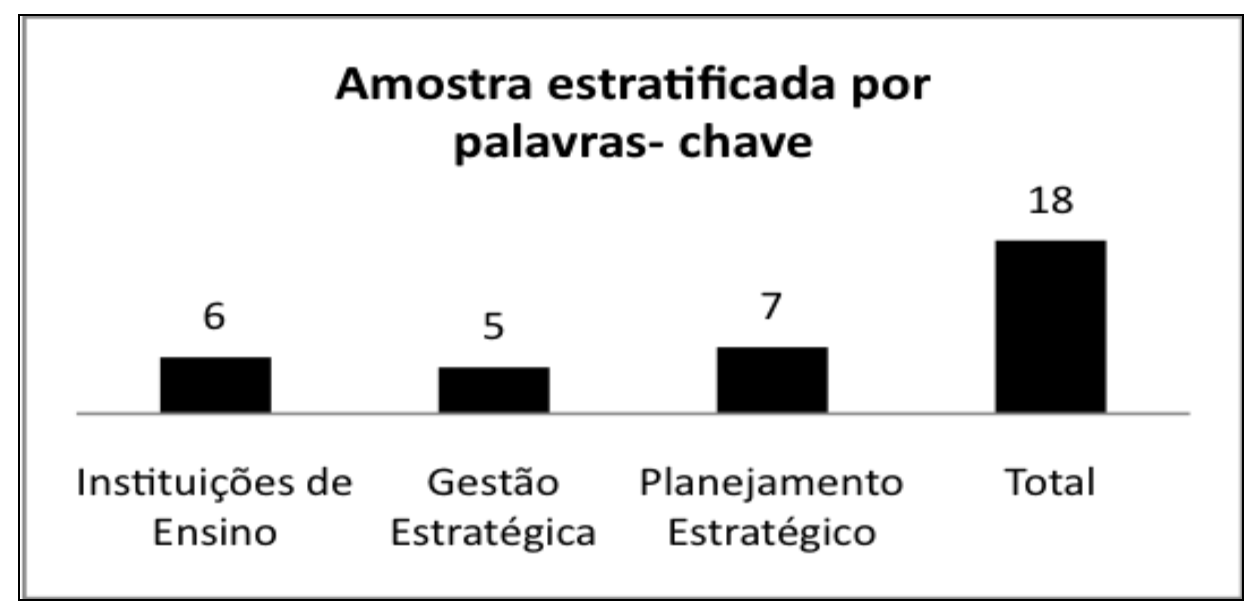

Gráfico 3 Amostra pesquisa estratificada por palavras-chave.

Fonte: Elaborado pelos autores (2012).

$\mathrm{Na}$ amostra pesquisada, identificou-se que 33\% dos artigos continham a palavra "Instituição de Ensino", 28\%, a palavra "Gestão Estratégica" e 39\%, a palavra "Planejamento Estratégico".

\subsection{CLASSIFICAÇÃO DOS ARTIGOS POR ANO DE PUBLICAÇÃO}

O Gráfico 4 apresenta a estratificação dos artigos da amostra pesquisada por ano de publicação.

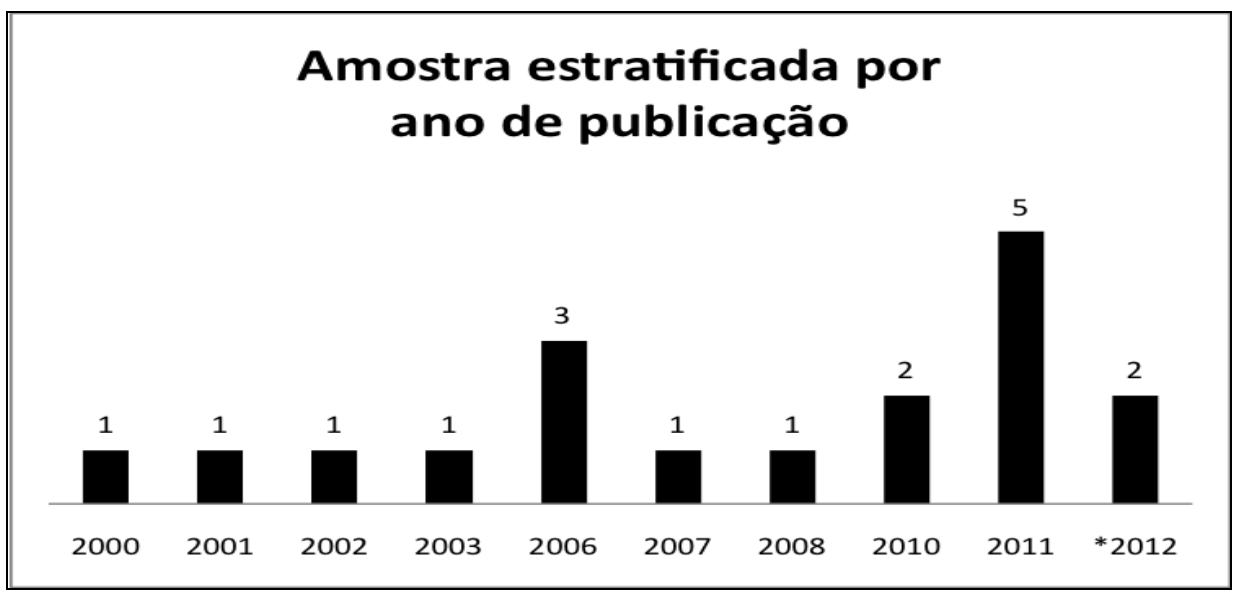

Gráfico 4 Amostra da pesquisa estratificada por ano de publicação.

Fonte: Elaborado pelos autores (2012). * Dados coletados até 15/06/2012. 
No Gráfico 4, destacam-se as produções realizadas nos anos de 2011, com cinco (5) artigos, e 2006, com três (3) artigos. Além disso, observa-se que 50\% (9) das publicações aconteceram no período de 2010 a 2012, concluindo-se a partir desta informação que se trata de um tema de discussão que recentemente vem ampliando-se.

\subsection{CLASSIFICAÇÃO DOS AUTORES MAIS PROLÍFICOS}

Para a definição dos autores mais prolíficos, levou-se em consideração o nome de todos os autores da amostra pesquisada. $\mathrm{Na}$ Tabela 3, visualizam-se todos os autores da amostra pesquisada:

Tabela 3 Autores da amostra pesquisada.

\begin{tabular}{|c|c|c|}
\hline ORDEM & $\begin{array}{r}\text { AUTORES } \\
\end{array}$ & QUANTIDADE DE ARTIGOS \\
\hline 1 & Leonardo Rocha de Oliveira & 2 \\
\hline 2 & Adriana Roseli Wünsch Takahashi & 1 \\
\hline 3 & Alberto G. Canen & 1 \\
\hline 4 & Aline de Oliveira Ferreira & 1 \\
\hline 5 & Alziro César de M. Rodrigues & 1 \\
\hline 6 & Amyra Moyzes Sarsur & 1 \\
\hline 7 & Auri Luiz Morais Rodrigues Filho & 1 \\
\hline 8 & Célia Maria Ribeiro & 1 \\
\hline 9 & Claudia Pedroso da Silva & 1 \\
\hline 10 & Dagmar Silva Pinto de Castro & 1 \\
\hline 11 & Denis Alcides Rezende & 1 \\
\hline 12 & Elenice Maria de Magalhães & 1 \\
\hline 13 & Eula Maria de Melo Barcelos Costa & 1 \\
\hline 14 & Fabia Maria de Souza & 1 \\
\hline 15 & Francisco José Lopes de Sousa Diniz & 1 \\
\hline 16 & Gabrielle Junqueira Hernandes & 1 \\
\hline 17 & Hélio Radke Bittencourt & 1 \\
\hline 18 & Hélio Raymundo Ferreira Filho & 1 \\
\hline 19 & Hernan Edgardo Contreras Alday & 1 \\
\hline 20 & Janete Lara de Oliveira Bertucci & 1 \\
\hline 21 & Janssen Edelweiss Nunes Fernandes Teixeira & 1 \\
\hline 22 & João Carlos da Cunha & 1 \\
\hline 23 & José Antonio Arantes Salles & 1 \\
\hline 24 & Juliano Aita Larán & 1 \\
\hline 25 & Leonardo Fabris Lugoboni & 1 \\
\hline 26 & Lúcia Mangolin & 1 \\
\hline 27 & Lucilaine Pascucci & 1 \\
\hline 28 & Marta Fabiano Sambiase Lombardi & 1 \\
\hline 29 & Milton Cordeiro Farias Filho & 1 \\
\hline 30 & Mírian Oliveira & 1 \\
\hline 31 & Peter Bent Hansen & 1 \\
\hline 32 & Regina Beatriz Bevilacqua Vieira & 1 \\
\hline 33 & Roberto Canquerini da Silva & 1 \\
\hline 34 & Rodrigo Scapini Meurer & 1 \\
\hline
\end{tabular}




\begin{tabular}{lll}
\hline & & \\
35 & Saul Eliahú Mizrahi & 1 \\
36 & Sérgio Feliciano Crispim & 1 \\
37 & Tatiane Monte Santana & 1 \\
38 & Telma Regina C. G. Barbosa & 1 \\
39 & Tereza Cristina Dias & 1 \\
40 & Vicente Garibotti & 1 \\
41 & Victor Meyer Jr. & 1 \\
42 & Zacarias Gonçalves de Oliveira Junior & 1 \\
43 & Zaki Akel Sobrinho & 1 \\
\hline Total & & $\mathbf{4 4}$ \\
\hline
\end{tabular}

Fonte: Elaborada pelos autores (2012).

Observa-se na amostra pesquisada uma dispersão no quesito autoria, o mesmo resultado foi encontrado em outras pesquisas que investigam o segmento das IES (CARPES, 2010; Corrêa, 2012).

\subsection{CLASSIFICAÇÃO DAS INSTITUIÇÕES DE ENSINO MAIS PROLÍFICAS}

Para a definiç̧ão das Instituições de Ensino mais prolíficas, levou-se em consideração o nome da Instituição referente ao primeiro autor do artigo. Na Tabela 4, visualizam-se as Instituições de Ensino mais prolíficas:

Tabela 4 Instituições de Ensino mais prolíficas.

\begin{tabular}{|c|c|c|c|c|}
\hline ORDEM & INSTITUIÇÕES DE ENSINO & SIGLA & $\mathbf{U F}$ & $\begin{array}{l}\text { QUANTIDADE } \\
\text { DE ARTIGOS }\end{array}$ \\
\hline 1 & $\begin{array}{l}\text { Pontifícia Universidade Católica do Rio Grande } \\
\text { do Sul }\end{array}$ & $\mathrm{PUC} / \mathrm{RS}$ & $\mathrm{RS}$ & 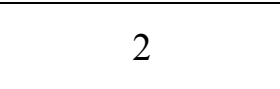 \\
\hline 2 & Pontifícia Universidade Católica do Paraná & PUC/PR & PR & 2 \\
\hline 3 & Universidade Federal do Paraná & UFPR & PR & 2 \\
\hline 4 & $\begin{array}{l}\text { Fundação Escola de Sociologia e Política de São } \\
\text { Paulo }\end{array}$ & FESPSP & SP & 1 \\
\hline 5 & Fundação Getúlio Vargas & $\mathrm{FGV} / \mathrm{SP}$ & SP & 1 \\
\hline 6 & $\begin{array}{l}\text { Pontifícia Universidade Católica de Minas } \\
\text { Gerais }\end{array}$ & $\mathrm{PUC} / \mathrm{MG}$ & $\begin{array}{l}M \\
G\end{array}$ & 1 \\
\hline 7 & Universidade de Trás-os-Montes e Alto Douro & UTAD & $*$ & 1 \\
\hline 8 & Universidade Federal da Bahia & UFBA & BA & 1 \\
\hline 9 & Universidade Federal de Goiás & UFG & GO & 1 \\
\hline 10 & Universidade Federal de Viçosa & UFV & DF & 1 \\
\hline 11 & Universidade Federal do Rio de Janeiro & UFRJ & $\mathrm{RJ}$ & 1 \\
\hline 12 & Universidade Federal do Rio Grande do Sul & UFRS & $\mathrm{RS}$ & 1 \\
\hline 13 & Universidade Metodista de São Paulo & UMESP & SP & 1 \\
\hline 14 & Universidade Municipal de São Caetano do Sul & USCS & SP & 1 \\
\hline 15 & Não identificado $* *$ & - & - & 1 \\
\hline Total & & & & 18 \\
\hline
\end{tabular}

Fonte: Elaborada pelos autores (2012). * Universidade de Portugal

** Não foi identificado o nome das instituições dos pesquisadores deste artigo. 
Na Tabela 4, destacam-se as Universidades PUC/RS, PUC/PR e UFPR com 6 artigos. Juntas, estas Universidades contribuem com 33\% das publicações da amostra, indo ao encontro dos achados de vários autores (LORDSLEEM, 2009; PIZZANI et al., 2010; CARPES, 2010; Corrêa, 2012; FILHO, 2012; SILVA et al., 2013), que apontam o sul e o sudeste do Brasil como as regiões mais prolixas na investigação de temas relacionados às IES.

\subsection{CLASSIFICAÇÃO DOS ESTADOS MAIS PROLÍFICOS}

Para a definição dos Estados mais prolíficos, levou-se em consideração o nome da instituição referente ao primeiro autor do artigo. Na Tabela 5, visualizam-se os Estados mais prolíficos:

Tabela 5 Estados mais prolíficos.

\begin{tabular}{clc}
\hline ORDEM & \multicolumn{1}{c}{ ESTADOS } & QUANTIDADE DE ARTIGOS \\
\hline 1 & Paraná & 4 \\
2 & São Paulo & 4 \\
3 & Rio Grande do Sul & 3 \\
4 & Minas Gerais & 1 \\
5 & Bahia & 1 \\
6 & Distrito Federal & 1 \\
7 & Goiás & 1 \\
8 & Rio de Janeiro & 1 \\
9 & $*$ Vínculo com universidade do exterior & 1 \\
10 & $* *$ Não identificado & 1 \\
\hline Total & & $\mathbf{1 8}$ \\
\hline
\end{tabular}

Fonte: Elaborada pelos autores (2012). * Universidade de Portugal

** Não foi identificado o Estado das instituições dos pesquisadores deste artigo.

Destacam-se os Estados do Paraná e São Paulo, com quatro (4) artigos cada, seguidos pelo Estado do Rio Grande do Sul, com três (3) artigos. Juntos, estes Estados representam $61 \%$ das publicações da amostra.

\section{CONSIDERAÇÕES FINAIS}

O estudo de temas como planejamento estratégico e gestão estratégica desperta o interesse dos pesquisadores. Apesar de ser um tema em discussão há mais de um século, ele continua sendo atual. Trata-se de uma tentativa de explicar e buscar uma forma de dirigir as organizações de forma competitiva (SERRA, 2007).

O objetivo desta pesquisa bibliométrica foi analisar os artigos publicados nos anais dos eventos acadêmicos da Associação Nacional de Pós-Graduação em Administração (ANPAD) 
e nos periódicos da Scientific Electronic Library Online (SciELO), no período de 1997 a 2012, referentes à produção e à disseminação do conhecimento científico sobre a gestão estratégica em Instituições Educacionais.

Constatou-se que o interesse em pesquisar o planejamento e a gestão estratégica das IES vem evoluindo, uma vez que se observa que 50\% (9) das publicações aconteceram no período de 2010 a 2012, sugere-se que esse aumento tem relação direta com o aumento da competitividade entre as IES, fator que motiva os agentes envolvidos neste meio a buscarem formas de se diferenciar, sendo que para isso faz-se necessária a utilização de ferramentas estratégicas para a gestão das IES.

Apesar do aumento das publicações nos últimos 2 anos investigados, percebeu-se uma dispersão com relação à autoria dos artigos. Entretanto, as Universidades PUC/RS, PUC/PR e UFPR são as mais prolíficas no tema. Juntas, estas Universidades contribuem com 33\% das publicações da amostra.

Como limitações da pesquisa, entende-se que: a leitura preliminar da população aconteceu somente no título e no resumo dos artigos, o que pode ter restringido o número da amostra; o baixo número de periódicos analisados; foram pesquisados apenas os artigos científicos disponibilizados na Internet; os artigos em língua estrangeira não fizeram parte da amostra; e a busca das terminologias supracitadas restringiu-se ao título, ao resumo e às palavras-chave.

Para futuras pesquisas, recomenda-se uma ampliação do escopo de pesquisa para outros periódicos bem como a utilização de ferramentas estatísticas que possibilitem uma maior compreensão entre as variáveis analisadas.

\section{REFERÊNCIAS}

ALVARENGA, Lídia. Bibliometria e arqueologia do saber de Michel Foucault: traços de identidade teórico-metodológica . Ciência da Informação, Brasília, v.27, n.3, p.1-9, 1998.

ANSOFF, H. I. Corporate Strategy, McGraw-Hill, New York, 1965.

ARAÚJO, Carlos Alberto. Bibliometria: evolução histórica e questões atuais. Em Questão, porto Alegre, v. 12, n. 1, p. 12-16, jan./jun. 2006.

CAMPOS, Ivan Ferreira de; VERNI, Marli de Lourdes. Gestão Universitária: Uma Analogia do Ponto de Vista de Dinossauros, Gazelas e Tigres em Três Instituições de Ensino Superior Privado. VI COLÓQUIO INTERNACIONAL SOBRE GESTÃO UNIVERSITÁRIA NA AMÉRICA DO SUL, Blumenau. Anais... Blumenaus, 15 a 17 Novembro, 2006. 
CHANDLER, A. D. Strategy and Structure: Chapters in the History of the American Industrial Enterprise. Cambridge, MA: The MIT Press, 1962.

CUNHA, Adriano Sérgio; SCHEFFEL, Fábio Roque; DUTRA, Ademar; LEITE, Andre Luis da Silva. Planejamento estratégico: mapeamento das publicações no período de 2000 a 2010. RPCA * Rio de Janeiro * v. 5 , n. 3, set./dez. 2011.

COLLIS, Jill; HUSSEY, Roger. Pesquisa em administração. Porto Alegre: Bookman, 2005.

CORRÊA, Guilherme Torres. Formação pedagógica no ensino superior: um olhar sobre os mestrados e os doutorados acadêmicos em Saúde Coletiva. 2012. Tese de Doutorado. Dissertação (Mestrado)-Programa de Pós-Graduação em Educação em Ciências e Saúde, Núcleo de Tecnologia Educacional para a Saúde, Universidade Federal do Rio de Janeiro, Rio de Janeiro, 2012.

DE MOURA CARPES, Aletéia et al. Uma Análise da Produção Científica Brasileira sobre Educação À Distância. VII Simpósio de Excelência em Gestão e Tecnologia. 2010.

ESTRADA, Rolando Juan Soliz; ALMEIDA, Martinho Isnard Ribeiro de . A eficiência e a eficácia da gestão estratégica: do planejamento estratégico à mudança organizacional. Revista de Ciências da Administração (CAD/UFSC), v. 9, p. 147-178, 2007.

ESPARTEL, Lélis Balestrin et al. Co-Autoria em Ensino e Pesquisa em Administração e Contabilidade no Brasil: Uma Década em Análise. Revista de Administração IMED, v. 1, n. $1,2011$.

HAMEL, Gary, PRAHALAD, C. K. Competindo pelo futuro. Rio de Janeiro: Campus, 1995.

HERNANDES, Gabrielle Junqueira e LOMBARDI, Marta Fabiano Sambiase. Um Estudo sobre o Processo de Gestão Estratégica em Instituições de Ensino Superior. V Encontro de Estudos em Estratégia - 3Es 2011 - Porto Alegre - RS.

HOUAISS, Antônio. Houaiss: Dicionário Eletrônico da Língua Portuguesa. São Paulo: Editora Objetiva Ltda, v. 1, 2009.

HUSSEY, D. Strategic management: from theory to implementation. Butterwood and Heinemann, London, 1998, 704p.

INSTITUTO NACIONAL DE ESTUDOS E PESQUISAS EDUACIONAIS. Censo da educação superior 2010. Brasília, 2011, 24p.

JR, Victor Meyer; PASCUCCI, Lucilaine; MANGOLIN, Lúcia. Gestão estratégica: um exame de práticas em universidades privadas. RAP — Rio de Janeiro 46(1):49-70, jan./fev, 2012 
JÚNIOR, Francisco Antonio Coelho. Gestão estratégica: um estudo de caso de percepção de mudança de cultura organizacional. Psico-USF, v.8, n.1, p. 81 a 89, Jan. a Jun. 2003.

JÚNIOR, Zacarias Gonçalves de Oliveira. CASTRO, Dagmar Silva Pinto. Gestão estratégica da tecnologia de informação em uma instituição de ensino como diferencial competitivo. $30^{\circ}$ Encontro da ANPAD - EnANPAD 2006 - Salvador - BA.

KNEIPP, J. M., PERLIN, A. P., de Moura Carpes, A., BEURON, T. A., XAVIER, T. R., \& JÚNIOR, V. F. S. Uma análise da produção científica brasileira sobre educação a distância. Revista Estudos do CEPE, Santa Cruz do Sul, n35, p.317-344, jan./jun. 2012.

LORDSLEEM, N., Araújo, R., Oliveira, B., \& Alexandre, M.. Ensino e pesquisa em administração: um estudo bibliométrico de publicações do EnANPAD (2001-2008). Revista Ciências Administrativas, 15(2), 356-378, 2009.

MACIAS-CHAPULA, Cesar A. O papel da informetria e da cienciometria e sua perspectiva nacional e internacional. Ciência da informação, v. 27, n. 2, p. 134-140, 1998.

MARCO, Ricardo Antônio de. O Processo de formação de estratégias em instituições de ensino superior.2011. Mestrado em Administração da Universidade do Sul de Santa Catarina, Florianópolis. 215p.

MINTZBERG, H. An Emerging Strategy of "Direct" Research. In: VAN MAANEN, John. Qualitative Methodology. California: Sage, 1983.

Generic strategies: toward a comprehensive framework. Advances in Strategic Management, v. 5, p. 01-67, 1988.

MINTZBERG, H. ; AHLSTRAND, B.; LAMPEL, J. Safári de estratégia: um roteiro pela selva do planejamento estratégico. Porto Alegre: Bookman, 2000.

MINTZBERG, H.; QUINN, J.B. The strategy process. Prentice Hall, 1992.

MINTZBERG, H.; McHUGH, A. Strategy formation in an adhocracy. USA: Administrative Science Quarterly, Jun, 1985.

NICOLAU, I. O conceito de estratégia. INDEG/ISCTE - Instituto para o desenvolvimento da Gestão Empresarial: Campo Grande, 2001.

PINTO, Francisco; LOIOLA, Elizabeth. Comportamento das firmas em redes de produção. GESTÃO. Org-Revista Eletrônica de Gestão Organizacional, v. 5, n. 1, 2010.

PIZZANI, Luciana; BELLO, Suzelei Faria; HAYASHI, Maria Cristina Piumbato Innocentini. Caracterização da produção científica em Educação Especial na Biblioteca Virtual em Saúde (BVS): um estudo bibliométrico. Revista Educação Especial, v. 23, n. 38, p. 379-397, 2010. 
REBELO, Luiza Maria Bessa; ERDMANN, Rolf Hermann. Modelo de Formação de Estratégias de Gestão Em Instituições de Ensino Superior sob o Enfoque da Teoria da Complexidade. RAI-Revista de Administração e Inovação, v. 4, n. 1, p. 05-20, 2007.

RICHARDSON, Roberto Jarry; DE SOUSA PERES, José Augusto. Pesquisa social: métodos e técnicas. Atlas, 1999.

ROBBINS, P. Greening the corporation. London: Earthscan Publication, 2001.

ROCHA, Daniela Torres et al. Processo de formação de estratégia em pequenas empresas: um estudo bibliométrico e sociométrico. Revista da Micro e Pequena Empresa, v. 5, n. 1, p. 102-119, 2011.

SANTOS, A. J. Robalo. Gestão Estratégica: Conceitos, modelos e instrumentos. Lisboa: Escolar editora, 2008.

SERRA, Fernando. Os pilares da estratégia: Estudo de caso de cinco empresas líderes. Revista Portuguesa e Brasileira de Gestão, v. 6, n. 2, p. 70-81, 2007.

STALLIVIERI, Luciane. O Sistema de Ensino Superior do Brasil: características, tendências e perspectivas. Acessoria de Relacões Interinstitucionais e Internacionais, UCS, 2006. Disponível em : http://bit.ly/oVfGas . Acesso em 02 de Junho de 2013.

SILVEIRA, N. P.;BASTIAS, A. P. Desafios e oportunidades: o verdadeiro papel do líder. Porto Alegre: SEBRAE/RS, 2003.

SILVA, Rosemary. Um estudo bibliométrico da produção científica no campo da Educação Especial. Revista Educação Especial, v.21, n.31, 2013.

VANTI, Nadia Aurora Peres. Da bibliometria à webometria: uma exploração conceitual dos mecanismos utilizados para medir o registro da informação e a difusão do conhecimento.

Ciência Informação, Ago 2002, vol.31, no.2, p.369-379.

VILAN FILHO, Jayme Leiro; ARRUDA, Raíza Veloso; PERUCCHI, Valmira. Análise das citações aos periódicos científicos brasileiros das áreas de Informação. Em Questão, Porto Alegre, v. 18, Edição Especial, dez. 2012. Disponível em:

$<$ http://seer.ufrgs.br/EmQuestao/article/view/33254>. Acesso em: 13

julho. 2013

YOSHINO, M.Y. Alianças estratégicas: uma abordagem empresarial a globalização. São Paulo, Ed. Makron Books, p. 288, 1997. 